This item was submitted to Loughborough's Research Repository by the author.

Items in Figshare are protected by copyright, with all rights reserved, unless otherwise indicated.

\title{
Experimental investigation of the aquatic propulsion caused by localised flexural wave propagation in immersed wedges and plates
}

PLEASE CITE THE PUBLISHED VERSION

http://dx.doi.org/10.1016/j.apacoust.2005.11.010

PUBLISHER

(C) Elsevier

VERSION

AM (Accepted Manuscript)

LICENCE

CC BY-NC-ND 4.0

\section{REPOSITORY RECORD}

Krylov, Victor V., and Gareth V. Pritchard. 2012. "Experimental Investigation of the Aquatic Propulsion Caused by Localised Flexural Wave Propagation in Immersed Wedges and Plates". figshare. https://hdl.handle.net/2134/11013. 
This item was submitted to Loughborough's Institutional Repository (https://dspace.lboro.ac.uk/) by the author and is made available under the following Creative Commons Licence conditions.

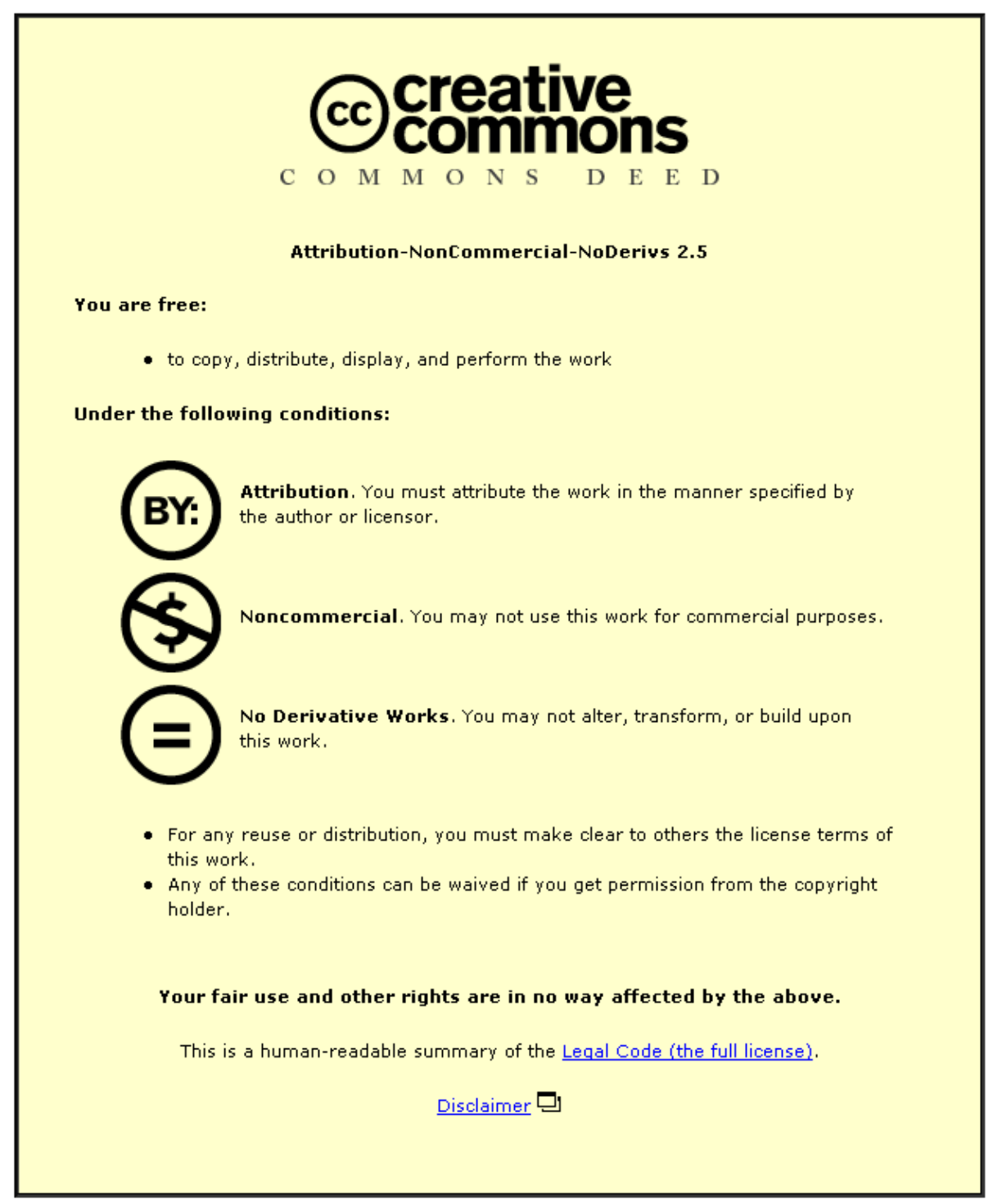

For the full text of this licence, please go to: http://creativecommons.org/licenses/by-nc-nd/2.5/ 


\title{
Experimental investigation of the aquatic propulsion
}

caused by localised flexural wave propagation in immersed

\author{
wedges and plates
}

\author{
V.V. Krylov and G.V. Pritchard \\ Department of Aeronautical and Automotive Engineering, \\ Loughborough University, \\ Loughborough, Leicestershire LE11 3TU, UK
}

\begin{abstract}
This paper describes the results of the experimental verification of the idea of wave-like aquatic propulsion of autonomous and man-inhabited vessels first published about 10 years ago by one of the present authors (V.V.K.). The idea is based on employing the unique type of localised flexural elastic waves propagating along edges of wedge-like structures immersed in water (wedge elastic waves). Such wedge-like structures supporting localised elastic waves can be attached like fish fins to a body of a small ship or a submarine and used for their propulsion. The proposed principle of employing localised flexural waves as a source of aquatic propulsion has been biologically inspired by the specific swimming mode used in nature by stingrays. To verify the idea experimentally, the first working prototype of a small catamaran using the above-mentioned wave-like propulsion via the attached rubber keel has been build and tested in Loughborough University. The tests have been carried out in two
\end{abstract}


phases, in a water tank and then in open water. The test results have shown that the catamaran was propelled very efficiently and could achieve the speed of $36 \mathrm{~cm} / \mathrm{s}$, i.e. about one vehicle length per second, thus demonstrating that the idea of wave-like propulsion of small maninhabited craft is viable. The reported proof of the viability of this idea may open new opportunities for marine craft propulsion, which can have far reaching implications.

Keywords: Wave-like aquatic propulsion; Localised wedge elastic waves; Small marine craft; Autonomous underwater vehicles; Experimental testing.

\section{Introduction}

The idea of wave-like aquatic propulsion of autonomous and man-inhabited vessels has been first published by one of the present authors [1] (see Fig. 1). This idea is based on employing the unique type of localised flexural elastic waves propagating along edges of wedge-like structures placed in contact with water (wedge elastic waves). Such wedge-like structures supporting localised elastic waves can be attached to a body of a small ship or a submarine like fish fins and used for aquatic propulsion. The above-mentioned wedge elastic waves propagating in contact with water have been first predicted and investigated in papers $[1,2]$ and later confirmed in the independent experiments $[3,4]$.

The principle of using localised flexural waves as a source of aquatic propulsion is similar to that used in nature by some fish, e.g. stingrays, which utilise wave-like motions of their large horizontal pectoral fins (wings) for moving forward. Note that there is a long history of human efforts to imitate fish swimming in man-made marine craft, especially in autonomous 
underwater vehicles (AUV) that are now being used in a wide variety of scientific investigations and surveillance operations [5-11]. Different types of fish swimming modes have been tried and copied extensively with a limited success. For example, the beating of the caudal fin or tail for propulsion, which is used most extensively in nature, has been subjected to a great deal of engineering imitations using simple oscillators or smart actuators (see e.g. [5-7, 9,10]). However, caudal fin type of propulsion, although applicable to AUV, is absolutely unsuitable for man-inhabited marine vessels, as the main body of the vessel would be rocked from side to side in reaction to the propulsion, making onboard conditions unpleasant and unsustainable.

For the above reason, the only mode of fish swimming which seems to be attractive for man-inhabited vessels is the undulatory wave-like motion seen in stingrays and skates. Stingrays and skates have large triangular pectoral fins (wings) that extend over the entire length of their body. The type of propulsion used by these fish is called 'rajiform' [10]. Manta rays swim in a similar way, but they flap their 'wings,' producing less than a single wavelength in the fin. Stingrays produce waves with smaller wavelength that propagate along the fin, accommodating up to three complete wavelengths along the fin length. It is this specific undulatory swimming mode used by stingrays that is inspirational and the most close to the wave-like propulsion employing localised wedge elastic waves described in the present work. It is vitally important for the application of wedge elastic waves for propulsion of maninhabited vessels that, in spite of the wings vibration, the main body of the craft remains virtually quiet because the energy of localised elastic waves is concentrated near the wings' tips [1].

The expected advantages of the new wave-like propulsion method of marine craft over the existing ones, e.g. jets and propellers, are the following: 
1. It is quiet, which is a particularly attractive feature for surveillance operations and for applications where minimal disturbance of wildlife is important;

2. It is energy-efficient since it follows nature (this, however, needs to be proven both theoretically and experimentally);

3. It is environmentally friendly and safe for people and wildlife.

Envisaged applications of wave like propulsion are small and medium research and pleasure man-inhabited surface ships and submersibles. Obviously, it can be used also for AUV propulsion, in addition to existing methods. Another possible application is sailing. One of the problems associated with sailing boats is that they are dependent on the wind for propulsion and are therefore stranded in very calm conditions. This is usually overcome with outboard motors used in times of low wind. However, in addition to being dead weight when not in use, these motors are usually placed at the extreme aft of the hull and can therefore cause an undesirable pitch to the boat. The replacement of the outboard motor by a flexible keel supporting localised flexural wave propagation and the associated wave-like propulsion, in place of the centre/dagger board, would have eliminate this problem. In particular, the centre of gravity of the propulsive system would be in the centre of the craft and in a very low position, giving the hull a greater stability. Apart from this, the propulsive keel (fin) would not interfere with the hydrodynamic characteristics of the hull or produce any additional drag as it simply replaces the already present component.

In the present paper, we report the results of the first experimental investigation of the above-mentioned idea of wave-like aquatic propulsion utilising localised flexural waves propagation in immersed elastic wedges and plates. To verify the idea experimentally, we have built and tested the first working prototype of a small catamaran employing the abovementioned wave-like propulsion via the attached rubber keel. The tests have been carried out in two phases, in a water tank and then in open water. The test results have shown that the 
catamaran was propelled very efficiently and could achieve the speed of about one its body length per second $(36 \mathrm{~cm} / \mathrm{s})$, thus demonstrating that the idea of wave-like propulsion of maninhabited craft is viable. The reported proof of the viability of this idea may open new opportunities for marine craft propulsion, which can have far reaching implications.

\section{Some properties of localised flexural waves in contact with water}

The wave-like propulsion of man-inhabited marine vessels, which is the main focus of this paper, is based on employing the unique type of localised flexural waves propagating along tips of elastic wedges submerged in water. Such waves, that are often called water-loaded wedge elastic waves, have been first predicted theoretically by one of the present authors (V.V.K.) in the same paper [1] that suggested the idea of using these waves for aquatic propulsion. Further developments of the theory of water-loaded wedge waves have been published in the paper [2], and their existence has been confirmed experimentally by independent researchers $[3,4]$.

Because of their complex nature, wedge waves generally can be described only numerically even for the simplest case of wedges in vacuum which was first considered back in the 1970's (see e.g. the book [12] and references there for more detail). This is even more so for wedges in contact with water. However, for the important case of slender wedges the situation can be simplified in both cases by using the geometrical acoustics approximation [12-15]. Using this approximation, one can solve the equation for bending vibrations of a slender wedge considered as a thin plate of variable thickness, thus obtaining relatively simple solutions for phase velocities of wedge waves and for their displacement amplitudes. Another significant advantage of the geometrical-acoustics approach is that it facilitates extending the analysis to more complex shapes of wedges (in addition to the simplest case of the so-called 
'linear' wedges that are formed by two intersecting planes) [15]. As a result, one can obtain relatively simple and physically explicit solutions for localised waves propagating in wedges in contact with vacuum [12-15] as well as in wedges immersed in water $[1,2,16,17]$. Other known approaches to analysing wedge elastic waves in contact with water include WienerHopf techniques [18] and finite element calculations [19].

For the purpose of aquatic propulsion, one can use wedge waves propagating in wedges of any shapes. The most suitable, however, appear to be quadratic wedges, which local thickness is described by the function $h(x)=\varepsilon x^{2}$, where $x$ is the distance from the edge and $\varepsilon$ is a constant. In such wedges all modes of localised flexural waves are dispersive, i.e. their phase velocities depend on frequency. This would allow an operator of a marine vessel with wavelike propulsion to change wedge wave velocity by changing frequency, which may be very convenient for efficient start and acceleration of the vessel from rest. Note in this connection that from the theory of swimming of slender fish, e.g. eels, it is known that the velocity of wave-like flexural motion of a fish body at stationary conditions should be slightly higher than the velocity of swimming [20-23]). Apart from this, the advantage of quadratic wedges is that they utilise a larger proportion of their surfaces for localised wave propagation in comparison with linear wedges, which again is beneficial for aquatic propulsion. From the point of view of practical implementation of wedge-like propulsive fins, it is also useful that phase velocities of localised flexural waves in quadratic wedges are very sensitive to edge truncations $[15,16]$. This allows such wedges to support waves with very low phase velocities. For example, for real-size immersed wedges made of rubber, wave velocities can be as low as a few meters per second, which is comparable with real speeds of swimming employed by marine craft.

Note that, although flexural waves in quadratic rubber wedges seem to be the most appropriate for aquatic propulsion of small man-inhabited craft, it was rather difficult to use 
such waves in the present experimental investigation due to difficulties in manufacturing of experimental wedges of quadratic shape. To pass by such difficulties in the preparation stage of these experiments, it was decided to avoid using localised flexural waves in quadratic wedges and to replace them by similar type of localised flexural waves propagating in simpler structures. In particular, one of the possibilities was to use the earlier established similarity between localised wave propagation in quadratic wedges and in the geometrically simpler systems comprising of thin elastic ridges embedded into an elastic half-space [15]. As it was shown in [15], localised flexural waves in quadratic wedges and in systems comprising of ridges embedded into an elastic half-space follow the same physical mechanisms of wave propagation and show similar dispersion behaviour.

From the point of view of flexural wave propagation, the latter systems in turn are similar to even more simple elastic structures - elastic strips with one free edge and with another edge being clamped. Note that all of the above-mentioned systems are characterised by similar dispersion behaviour of localised waves caused by physical similarity in waveguide properties of such systems. In particular, there are different modes of flexural waves in each system, which all have minima of phase velocity at certain frequencies. Therefore, for experimental purposes of this paper, it was more practical to use 'clamped-free' rubber plates instead of ideally suitable quadratic rubber wedges or elastic ridges embedded into an elastic half-space. One should keep in mind however that, in contrast to quadratic wedges, such 'clamped-free' plates do transmit vibrations to the main body of a vessel through the area of clamping. Therefore, although quite suitable for AUV, the aforementioned 'clamped-free' rubber plates can not be recommended for applications to real man-inhabited marine vessels. Nonetheless, for the purpose of experimental investigations on a model vessel described in this paper they are perfectly acceptable. 
Note that all of the above-mentioned localised flexural waves in contact with water are waves propagating in the subsonic regime of wave propagation (in respect of underwater sound). As it is well known, such waves ideally do not generate sound in the surrounding water. Therefore, the associated aquatic propulsion is virtually quiet, which is an attractive feature for both man-inhabited vessels and AUV. In the latter case, a significant attention is currently being paid to decreasing the radiated noise [8]. Note that the above-mentioned localised flexural waves that are used in the experimental part of this work are guided modes of a finite strip, and they are different from the so-called localised flexural waves of Rayleigh type propagating along edges of semi-infinite plates (see e.g. [24]).

Figure 2 shows the theoretically calculated frequency-dependent phase velocities (dispersion curves) of the lowest-order flexural mode in an immersed 'clamped-free' rubber strip. Calculations have been carried out using the geometrical acoustics approach [1,2] for the two values of strip thickness: $1 \mathrm{~mm}$ and $3 \mathrm{~mm}$. The width of the strip was $150 \mathrm{~mm}$ in both cases. For comparison, the corresponding phase velocities of flexural waves in infinite immersed plates of the same thickness are shown as well. One can see that for a strip of $1 \mathrm{~mm}$ thickness the dispersion curves for a finite strip are almost indistinguishable from the dispersion curves for an infinite plate of the same thickness that does not support waveguide flexural wave propagation. For a $3 \mathrm{~mm}$ strip the phase velocity is slightly higher than in an infinite plate of the same thickness and has a minimum at frequency of around $0.3 \mathrm{~Hz}$.

Note that in the case under consideration the calculated phase velocities of flexural waves in immersed 'clamped-free' rubber strips are very low, from about 2 to $45 \mathrm{~cm} / \mathrm{s}$, depending on frequency (these are much lower than wave velocities in the same strips and plates in vacuum). Such low wave velocities have been specifically chosen to provide several wavelengths of flexural waves on the length of the propulsive fin, which was required to emulate the 'rajiform' wave-like motion associated with stingrays. 


\section{Design of the model vessel and of the experimental rig}

According to the previous section, in the framework of this paper to produce wave-like propulsion of a small model of a marine vessel it was sufficient to use an elastic rubber plate clamped on one side, instead of using a quadratic rubber wedge. The plate was to be excited by given flexural displacement at a single point, with the resulting flexural waves propagating in the direction opposite to the direction of swimming. Although the main envisaged applications for the wave-like propulsion considered is for use on small ocean man-inhabited research submarines and AUV, at this early stage of the investigation the submarine design was not implemented. The main reasons for that were difficulties in sealing the craft and the associated unnecessary complications that would make any experimental modifications more difficult. Instead, a simpler surface-boat-type vessel (a catamaran) and the associated experimental rig have been designed and built.

The basic rig comprised of the central propulsive plate (fin) mounted on the two supporting pylons $(30 \mathrm{~cm}$ each) positioned at 90 degrees to the fin (see Fig. 3). The pylons could then either be clamped above a water tank to study water flows generated by the vibrating propulsive fin or their ends could be attached to a pair of pontoons to form a catamaran. The propulsive rubber plates were clamped along one edge and were free on the other three sides, thus forming the aforementioned 'clamped-free' strips of finite length. The plates were excited at the tip by given flexural displacements of a mechanical pivoted arm that was attached to the leading edge of the propelling plate and driven by the electric motor. The motor was connected to the exciting pivoted arm via an additional mechanical arm and a disk with a set of 12 drilled holes placed at different distances from the centre of rotation. The drilled holes allowed for a change in amplitudes of the plate excitation by changing the 
pivotal position of the additional mechanical arm. These holes were designated by the symbols D1 - D12, from innermost to outermost ones, each of them being associated with the specific increased value of the displacement amplitude of the plate tip. The whole mechanical system under consideration was thus used to transform the rotational movement of the electric motor to the translation movement of the exciting arm used for flexural wave generation.

The experimental testing took place in two stages. The first stage had been carried out in a water tank, where the rig (the propulsive part of the model vessel) did not move. This testing in an enclosed and controlled environment allowed for easier and more accurate observations of the water-loaded flexural waves generated in an immersed propulsive fin and of the resulting water flows associated with the propulsion. The second stage took place in open water, with the rig mounted on a catamaran and moving due to the effect of the wave-like propulsion.

The size and thickness of the propulsive plate and the range of driving frequencies were important parameters to be considered. Assuming that frequencies of wave excitation are in the range $2-10 \mathrm{~Hz}$, the thickness of the basic rubber plate was chosen as $1 \mathrm{~mm}$. The width (span) and the length (chord) of the propulsive rubber plate were chosen as $150 \mathrm{~mm}$ and 250 mm respectively.

A $12-24 \mathrm{~V}$ dc motor with a gearbox was chosen to power the craft. This motor was selected for its small size and relatively high torque. The gearbox ratio could be adjusted between 4:1 and 4096:1. In particular, using a 64:1 gear ratio gave a suitable maximum rotational speed of roughly $300 \mathrm{rpm}($ or $5 \mathrm{~Hz}$ ) at the maximum rated voltage of $24 \mathrm{~V}$. The clamping of the propulsive rubber plate was realised by means of a hollowed aluminium bar that contained a set of nuts and bolts on both sides of the rubber plate. 
A clear plastic water container was used for the in-tank testing. The twin pylons supporting the fin and motor were sized to fit by bolts exactly across the top of the container. Water was then added to the container to submerge the fin span.

The open-water stage of testing incorporated the same basic propulsive test rig as the water-tank testing, with the addition of buoyancy aids to the sides of the rig to assemble a catamaran craft. Two Styrofoam pontoons were placed between the two supporting plates in place of the sides of the water container. The complete open-water test rig (a catamaran) is shown in Fig. 4.

The pontoons were designed to maintain the fully immersed water depth of the fin. The rig was weighed, and the pontoons were sized accordingly. With the motor mass offset from centre in both the longitudinal and lateral directions, the right-hand pontoon needed to be longer than the left-hand $(50 \mathrm{~cm}$ and $35 \mathrm{~cm}$ respectively), with both pontoons extending forward of the main deck. This configuration ensured a level deck of the vessel.

\section{Testing in a water tank}

During the testing in a water tank the propulsive fin was placed vertically in the centre of the container. The flow speeds were measured by timing the movement of the small pieces of cork floated in water over a set distance. The scatter of the measured speeds was within $5 \%$, therefore all measurements were performed three times, and the results were averaged.

Several preliminary tests have been made in order to find the best test parameters. In particular, water levels of $1 / 3$ span, $2 / 3$ span, complete immersion and complete immersion plus $40 \mathrm{~mm}$ were examined. It was found that non-complete immersion resulted in choppy flow conditions, whereas complete immersion produced a less choppy surface flow. Complete immersion plus $40 \mathrm{~mm}$ had an even smoother surface with little decreased flow, but it raised 
the water too close to the electric motor. As a compromise, a barely fully immersed plate was used for all measurements.

The vertical position of the excitation point on the leading edge of the propulsive fin was also examined. It was found that positioning the excitation point at the tip to approximately $95 \%$ of the total span, which roughly corresponds to a maximum of the energy flow carried out by a localised flexural wave, resulted in the most efficient flexural wave generation and higher levels of propulsion.

To optimise the thickness of the propulsive fin, in addition to the basic plate of $1 \mathrm{~mm}$ thickness (see previous section), two thicker pieces of rubber of the same dimensions were tested. The thickest was of the order of $3 \mathrm{~mm}$ thick, and the thinner was approximately $2 \mathrm{~mm}$ thick. The above-mentioned three rubber samples will be referred to as thin (1mm), medium $(2 \mathrm{~mm})$ and thick $(3 \mathrm{~mm})$. As expected, for operating frequencies from 2.5 to $4.5 \mathrm{~Hz} \mathrm{a}$ complete flexural wavelength was not observed along the chord of the thick rubber plate, and the fin simply acted as a side-to-side paddle. On the other hand, the most flexible thin sample $(1 \mathrm{~mm})$ allowed for a number of wavelengths to be observed along the chord of the fin. The medium plate $(2 \mathrm{~mm})$ behaved similarly to the thin plate, but supported a fewer number of wavelengths along the chord for a given input frequency.

The main part of the testing in a water tank included observation of the behaviour of the fin as a source of propulsion over a range of amplitudes and frequencies. The frequency (in $\mathrm{Hz}$ ) was equal to a number of motor revolutions per second. The actual observed frequency depended on the loading and on the input electric power. The direct current power adapter was set up to allow the voltage to be user defined. The propulsive system was then preliminary tested in a tank at 1-volt intervals and the electric current was read off to determine the input electric power. Increasing the voltage increased the power and therefore increased the speed of the motor rotation and thus the frequency of the fin vibrations. It has 
been checked experimentally that the frequency of vibration versus power in from the motor was a straight line. In particular, over the whole range of power in used in the experiments, i.e. from 2 to 6 Watts, the frequency changed linearly from 2.5 to $4.5 \mathrm{~Hz}$. The speed of rotation, which was equal to the excitation frequency of fin vibrations, was measured using a reflective sensor and an electronic counter or oscilloscope. Results were recorded for 4 values of fin vibration amplitudes at 11 voltage settings, from $15 \mathrm{~V}$ to $25 \mathrm{~V}$ at 1 -volt intervals, changing the frequency of fin vibration. The input electric power and frequency were determined at these positions and plotted against the measured flow speed. The obtained results are shown on Figs. 5 and 6.

It can be seen that the speed of the flow increased with increasing frequency and amplitudes of vibration (see Fig. 5) as well as with increasing of the input electric power (Fig.6). The observed flow velocities in the water tank can be compared with the phase velocities of localised flexural waves propagating along the propulsive rubber fins. As one can see from Fig. 5, in the frequency range 2.4-4.5 Hz the experimental flow velocities varied from $1 \mathrm{~cm} / \mathrm{s}$ to $11 \mathrm{~cm} / \mathrm{s}$, depending on flexural wave amplitudes. According to the theoretical calculations of phase velocities of localised flexural waves propagating in 'clamped-free' water-loaded rubber strips of the same width and of the same thickness $(\mathrm{h}=1 \mathrm{~mm})$, these velocities varied from $12 \mathrm{~cm} / \mathrm{s}$ to $17 \mathrm{~cm} / \mathrm{s}$ (see Fig. 2). Thus, the observed velocities of the flows always made up a fraction of the corresponding wave phase velocities, increasing with the increase of the wave amplitudes.

It is interesting to note from Fig. 5 that minima on the plots corresponding to different vibration amplitudes occur at different frequencies. Apparently, the observed minima of flow speeds can be associated with the plate natural frequencies at which the localised flexural waves fail to propagate and form partial standing waves along the chord, thus displacing some amount of water at 90 degrees from the desired flow direction. The first natural frequency of 
the plate operated at the disc amplitude setting D8, which corresponds to the highest value of the fin tip displacement amplitude $\mathrm{U}_{0}=3.2 \mathrm{~cm}$, was at approximately $2.7 \mathrm{~Hz}$. The same plate excited at D2 setting (i.e at $\mathrm{U}_{0}=1.8 \mathrm{~cm}$ ) had its first natural frequency at $3.2 \mathrm{~Hz}$.

The observed dependence of the natural frequencies on vibration amplitudes can be explained by the presence of non-linear effects for localised flexural waves considered. Indeed, typical displacement amplitudes of generated flexural waves $(2.5 \mathrm{~cm})$ were large enough in comparison with their typical wavelengths $(8 \mathrm{~cm})$, resulting in the values of Mach number around two, so that non-linear effects must have been essential for the flexural waves considered. These non-linear aspects, however, would require a special investigation, which is out of the scope of this paper.

The input electric power readings were taken for each setting in order to estimate the size of battery required for testing in open water. Naturally, the best operating setting for sustained running of the model vessel in open water would be the one corresponding to the maximum efficiency of propulsion. Using the measured values of the input electric power and of the flow speed in a water tank, it was easy to estimate a crude value of the propulsive efficiency of the fin at the various test conditions. To do so one has to estimate the output power associated with the generated water flow. The latter can be calculated as the product of the associated thrust force produced by the fin $F$ and the flow velocity $v$.

$$
P_{\text {out }}=F v
$$

If the speed of the flow $v$ is constant over time, then the combined drag forces $D$ generated by the fin, container and all other surfaces must be equal to the thrust force $F$ produced by the fin. On the other hand, it is well known that the drag force $D$ is a function of a reference area $A$, mass density of the liquid $\rho$ and the square of flow velocity $v$ (see e.g. [25]): 


$$
D=(1 / 2) C_{D} A \rho v^{2}
$$

where $C_{D}$ is the drag coefficient of a system. Taking into account that $F=D$ and substituting Eqn (2) into (1), one can obtain the following expression for $P_{\text {out }}$ :

$$
P_{\text {out }}=B v^{3}
$$

where $B=(1 / 2) C_{D} A \rho=$ const. The efficiency $\eta$ can then be calculated as the power out of the fin divided by the power in to the fin:

$$
\eta=\frac{P_{o u t}}{P_{\text {in }}}=\text { const } \cdot \frac{v^{3}}{I \cdot V}
$$

As there is still an unknown constant in the equation (4) depending on the parameters of the rig, the expression (4) can be only used for relative comparison of the efficiencies for the same experimental rig, e.g. by normalising them with respect to the maximum efficiency achieved. No meaningful comparison can be made to any other system. The relative value of efficiency normalised in this way will be referred to as fraction of maximum efficiency.

Note that in the above-mentioned definition of efficiency, we did not take into account the dependence of the drag coefficient on amplitudes and wavelengths of flexural waves, assuming that the wave amplitudes are small enough, so that the drag is defined primarily by the body of the vessel. However, for waves of larger amplitudes, the amplitude-related effects associated with changes in the form and lateral dimensions of the fin are expected to influence the total drag coefficient. The situation here is similar to the one associated with non-linear wave effects resulting in the observed dependence of locations of minima in the generated 
flow velocities. Indeed, one could expect that the drag should increase for larger vibration amplitudes and for smaller wavelengths, i.e. in line with the increase of Mach numbers of propagating flexural waves. The character of this dependence is not clear, and it will be investigated in the future work.

The fraction of maximum efficiency as function of frequency is shown in Fig. 7. One can see that the values of efficiency generally rise with the increase of frequency; the natural frequencies are again well defined by the minima in the data. The maximum efficiency appears at around $3.8 \mathrm{~Hz}$, with the amplitude setting D6 $\left(\mathrm{U}_{0}=2.8 \mathrm{~cm}\right)$. The lowest efficiency, which is very close to zero, occurs at $3.3 \mathrm{~Hz}$ for the amplitude setting D2 $\left(\mathrm{U}_{0}=1.8 \mathrm{~cm}\right)$. The frequencies at which the highest and lowest efficiencies occur correspond to input voltages of $23 \mathrm{~V}$ and $18 \mathrm{~V}$ respectively.

To achieve the desired maximum efficiency for testing in open water the system would have to be pulling $5 \mathrm{~W}$ of electric power. If one used small electric batteries on board the vessel to power it in open water, this kind of power would have drained a set of batteries very quickly. Apart from this, this would add a lot of weight to the model vessel. The alternative was to use lead acid batteries for the open water testing. These were exceptionally heavy and required to be off the craft with a wire feed.

\section{Testing in open water}

The open-water testing was carried out in the experimental pool that could be considered as open water for the assembled small model catamaran with the wave-like propulsion. Although the open-water testing was designed mainly to prove the idea and not to gain accurate results, the craft nonetheless was tested in several configurations. Two sets of tests 
were performed. The first was a timed run over a distance of $3 \mathrm{~m}$, the craft starting form rest. This was designed to measure averaged speeds of the craft over this distance. The second set of tests was again a timed run over a distance of $2 \mathrm{~m}$, but with an initial speed achieved after passing the added 'acceleration' distance of $2 \mathrm{~m}$, an ample space to reach what appeared to be a stationary speed. This set of tests was obviously designed to measure stationary speeds.

The start and end positions were marked on the pool edge and on the pool bottom. In addition to the three black rubber plates tested in a water tank, a white rubber plate was added for open-water testing. This white rubber was of the same thickness as the thin black rubber, but was less stiff. As in the water-tank tests, a single rubber plate was used (1 sheet) with dimensions $250 \times 150 \mathrm{~mm}$, or one could use a rubber plate of the same size supported by two smaller side plates ( 3 sheets) with dimensions $70 \times 250 \mathrm{~mm}$.

When powered from the external batteries via a flexible cable, the catamaran demonstrated fast acceleration from rest to stationary speeds that were high enough in terms of its lengths per second (see Fig. 8). The results of the two sets of tests for different configurations are presented in Tables 1 and 2. They have been arranged in time order, the fastest at the top, with an averaged time (and the resulting averaged speed) obtained over the three runs. The amplitude settings were D4 $\left(\mathrm{U}_{0}=2.2 \mathrm{~cm}\right), \mathrm{D} 6\left(\mathrm{U}_{0}=2.8 \mathrm{~cm}\right)$ and $\mathrm{D} 8\left(\mathrm{U}_{0}=3.2 \mathrm{~cm}\right)$. The obtained results demonstrate that the wave-like aquatic propulsion considered is viable and efficient.

Note that the propulsive thin plate performed more efficiently when flanked by additional two smaller rubber sheets. A possible explanation for this could be that a sided plate, being thicker, has a higher wave velocity, thus resulting in a higher stationary swim speed.

The qualitative comparison of the measured steady craft speed in open water versus predicted phase velocities has shown that the achieved speeds were comparable with the wave speeds in the propulsive plate at the operating frequencies, around $3.8 \mathrm{~Hz}$. However, no 
detailed comparison with the predicted phase velocities has been made. The reason for that was that the experimental rubber fin used in open water testing was reinforced by two smaller pieces of rubber and wave speeds for such a system could not be calculated. One can expect, however, that these speeds were between the two theoretically predicted values of 13 and 31 $\mathrm{cm} / \mathrm{s}$, corresponding to a single rubber plate with thickness of 1 and $3 \mathrm{~mm}$ respectively (see Figure 2). Measuring actual wave velocities of localised flexural waves was out of the scope of this paper, and it will be carried out in the future work.

The comparison of the speeds of normal and heavier craft (with additional weight) has shown, as expected, that the normal craft performed better than the heavier one as it sat further out of the water and therefore produced less drag.

As expected, the results on propulsion efficiency obtained from the in-tank testing agreed with those observed in the open-water test. For example, at the open-water test condition of 22 volts, the most efficient amplitude setting was D6, then D8, and then D4, which was in agreement with the in-tank testing.

It would be interesting, of course, to compare the efficiency of the described wave propulsion with the efficiency of a propeller. However, such a comparison was beyond the scope of this paper concerned primarily with the feasibility studies. To make such a comparison meaningful one would require to optimise the mechanical design of the model vessel employing the proposed wave propulsion system. In the current design, which employs the motor disk and mechanical arms for flexural wave generation, a substantial amount of energy is being lost due to friction at the mechanical arm contact points. This reduces substantially the energy efficiency of the system. In the future design, an improved version of the model vessel could be developed, e.g. using electro-active bending polymers (EAP) to directly generate flexural waves at a leading edge in a plate or wedge, instead of the mechanical arms. This would reduce or even eliminate any moving parts from the exciting 
system. It is expected that energy efficiency of such an improved vessel would be essentially higher, and it would be possible to make its detailed comparison with vessels employing traditional propeller systems.

\section{Conclusions}

The first and most important conclusion resulting from this work is that localised wedge or plate flexural elastic waves can indeed be used to generate wave-like aquatic propulsion and to propel a small marine craft. To propel a man-inhabited craft one should use propulsive fins made of quadratic elastic wedges that keep the wave vibration energy away from the main body of the vessel.

A wave propulsion rig has been created that produces thrust both in the controlled watertank test environment and in the open water.

The results of the in-tank testing have shown that the speeds of wave-generated water flows depend on the amplitude and frequency of wave excitation as well as on the input electric power. The conditions have been established corresponding to the maximum efficiency of the aquatic propulsion. These conditions have then been used for testing of the model catamaran in open water.

In the open water testing, it has been shown that the propulsion is operable over a continuum of speeds, including acceleration from rest, and that its efficiency can be maximised by changing the amplitude and frequency of the input force.

The speeds achieved by the small model catamaran in the open water testing are comparable with the wave speeds in the propulsive plate at the operating frequencies. This is in agreement with the well-known results following from the studies of fish swimming 
indicating that speed of swimming at stationary conditions should be slightly less than the velocity of wave-like motion of the fish body.

Although the current experimental rig used a rather complex mechanical construction to achieve the localised flexural wave excitation, it is expected that further investigations could lead to the development of simpler and more efficient marine craft. In particular, this could include the use of electro-active bending polymers (EAP) to directly generate flexural waves at a leading edge in a plate or wedge, instead of the rather complex mechanical exciter used in this work. This would reduce or even eliminate any moving parts from a wave propulsion rig, giving it another advantage over a conventional propeller.

Further work is required to investigate the efficiency of wave propulsion in comparison with its main rival, a propeller. However, even if the efficiency of wave-like propulsion cannot be developed to surpass that of the propeller, there is still an unexplored niche for it. Namely, wave-like propulsion may have no rivals in cases where quiet and safe operation is paramount, in particular in the cases of small man-inhabited research submarines and autonomous underwater vehicles (AUV). 


\section{References}

[1] Krylov, V.V. Propagation of wedge acoustic waves along wedges embedded in water. Proc IEEE Ultrasonics Symposium, Cannes, France, 1994; 793-796.

[2] Krylov, V.V. On the velocities of localized vibration modes in immersed solid wedges. Journ. Acoust. Soc. Amer., 1998; 103: 767-770.

[3] Chamuel, J.R. Flexural edge waves along free and immersed elastic waveguides. Review of Progress in Quantitative Nondestructive Evaluation, Vol.16, ed. D.O. Thompson and D.E. Chimenti (Proc. 16th Symp. Quant. Nondestruct. Eval., July 28-August 2, 1996, Brunswick, Maine), Plenum Press, New York, 1996; 129-136.

[4] De Billy, M. On influence of loading on the velocity of guided acoustic waves propagating in linear elastic wedges. Journ. Acoust. Soc. Amer., 1996; 100: 659-662.

[5] Bandyopadhyay, P.R. Trends in biorobotic autonomous undersea vehicles. IEEE Journal of Oceanic Engineering, 2005; 30: 109-139.

[6] MacIver, M.A., Fontaine, E. and Burdick, J.W. Designing future underwater vehicles: principles and mechanisms of the weakly electric fish. IEEE Journal of Oceanic Engineering, 2004; 29: 651-659. 
[7] Shinjo, N. and Swain, G.W. Use of a shape memory alloy for the design of an oscillatory propulsion system. IEEE Journal of Oceanic Engineering, 2004; 29: 750-755.

[8] Zimmerman, R., D’Spain, G.L. and Chadwell, C.D. Decreasing the radiated acoustic and vibration noise of a mid-size AUV. IEEE Journal of Oceanic Engineering, 2005; 30: 179-187.

[9] Yamamoto, I., Terada, Y., Nagamatu, T. and Imaizumi, Y. Propulsion system with flexible/rigid oscillating fin. IEEE Journal of Oceanic Engineering, 1995; 20: 23-30.

[10] Sfakiotakis, M., Lane, D.M., and Davies, J.B.C. Review of fish swimming modes for aquatic locomotion. IEEE Journal of Oceanic Engineering, 1999; 24: 237-252.

[11] Guo, S., Fukuda, T. and Asaka, K. A new type of fish-like underwater microrobot. IEEE/ASME Transactions on Mechatronics. 2003; 8: 136-141.

[12] Biryukov, S.V., Gulyaev, Yu. V., Krylov, V.V. and Plessky, V.P. Surface Acoustic Waves in Inhomogeneous Media, Springer-Verlag, Berlin, Heidelberg, 1995.

[13] Krylov, V.V. Conditions for validity of the geometrical-acoustics approximation in application to waves in an acute-angle solid wedge. Soviet Physics - Acoustics, 1989; 35: 176-180.

[14] Krylov, V.V. Geometrical-acoustics approach to the description of localized vibrational modes of an elastic solid wedge. Soviet Physics - Technical Physics, 1990; 35: 137-140. 
[15] Krylov, V.V. Localized acoustic modes of a quadratically-shaped solid wedge. Moscow University Physics Bulletin, 1990; 45(6): 65-69.

[16] Krylov, V.V. and Shuvalov, A.L. Propagation of localised flexural vibrations along plate edges described by a power law. Proceedings of the Institute of Acoustics, 2000; 22(2): 263-270.

[17] Shuvalov, A.L. and Krylov, V.V. Geometrical-acoustics consideration of the flexural modes in immersed anisotropic wedges. Journ. Sound and Vibr., 2000; 237: 427-434.

[18] Shanin, A.V. and Krylov, V.V. An approximate theory for waves in a slender elastic wedge immersed in liquid. Proc. Roy. Soc. Lond. A, 2000; 456: 2179-2196.

[19] Hladky-Hennion, A.-C., Langlet, P., and de Billy, M. Finite element analysis of the propagation of acoustic waves along waveguides immersed in water. Journ. Sound and Vibr., 1997; 200: 519-530.

[20] Lighthill, M. J. Note on the swimming of slender fish, Journ. Fluid Mech., 1960; 9: 305-317.

[21] Lighthill, M. J. Aquatic animal propulsion of high hydrodynamic efficiency, Journ. Fluid Mech., 1970; 44: 265-301. 
[22] Lighthill, M.J. Large amplitude elongated-body theory of fish locomotion, Proc. Roy. Soc. Lond. B, 1971; 179: 125-138.

[23] Cheng, J.Y. and Blikhan, R. Note on the calculation of propeller efficiency using elongated body theory, Journ. Exp. Biol., 1994; 192: 169-177.

[24] Norris, A.N., Krylov, V.V and Abrahams, I.D. Flexural edge waves and Comments on "A new bending wave solution for the classical plate equation"[J. Acoust.Soc. Am. 104, 2220-2222 (1998)]. Journ. Acoust. Soc. Amer., 2000; 107: 1781-1784.

[25] Batchelor, G.K. An introduction to fluid dynamics. Cambridge University Press, Cambridge, 1994. 


\section{Figure legends}

Fig. 1. On the proposed use of wedge elastic waves for propulsion of a small submarine [1]; the localised flexural waves propagate along the tips of the horizontal fins (wings), their energy being concentrated away from the main body.

Fig. 2. Theoretical phase velocities of the lowest order flexural mode in two 'clamped-free' water-loaded rubber strips as functions of frequency; both strips are of the same width $H=150 \mathrm{~mm}$, but have different values of thickness: $h=1 \mathrm{~mm}$ (solid curve) and $h=$ $3 \mathrm{~mm}$ (dashed curve); for comparison, the velocities of flexural waves in infinite plates are also shown for $h=1 \mathrm{~mm}$ (dotted curve, which practically coincides with the solid curve) and for $h=3 \mathrm{~mm}$ (dash-dotted curve).

Fig. 3. View of the assembled propulsion rig in an empty water tank used for in-tank testing: the main propulsive plate made of white rubber is stiffened by two narrower plates of black rubber; holes drilled on the motor disk are used for changing amplitudes of plate vibration.

Fig. 4. View of the assembled catamaran with wedge wave propulsive system before its testing in open water. 
Fig. 5. Recorded speed $v$ of the flow generated in a water tank by a localised wedge elastic wave propagating along the rubber plate as function of frequency for different values of the wave displacement amplitudes; the amplitudes are identified by the locations of the holes on the motor disk: holes D2, D4, D6 and D8 correspond to the plate tip displacement amplitudes of 1.8, 2.2, 2.8 and $3.2 \mathrm{~cm}$ respectively.

Fig. 6. Recorded speed $v$ of the flow generated in a water tank by a localised wedge elastic wave propagating along the rubber plate as function of input electric power for different values of the wave displacement amplitudes; the amplitudes are identified by the locations of the holes on the motor disk: holes D2, D4, D6 and D8 correspond to the displacement amplitudes of 1.8, 2.2, 2.8 and $3.2 \mathrm{~cm}$ respectively.

Fig. 7. Fraction of maximum efficiency of the process of flow generation in a water tank by a localised wedge elastic wave propagating along the rubber plate as function of frequency for different values of the wave displacement amplitudes; the amplitudes are identified by the locations of the holes on the motor disk: holes D2, D4, D6 and D8 correspond to the displacement amplitudes of $1.8,2.2,2.8$ and $3.2 \mathrm{~cm}$ respectively.

Fig. 8. View of the moving catamaran with wedge wave propulsive system during its testing in open water. 


\section{List of tables}

Table 1. Open-water testing from rest

Table 2. Open-water testing with initial craft speed 


\section{Tables}

Table 1. Open-water testing from rest

\begin{tabular}{|c|c|c|c|c|c|c|}
\hline $\begin{array}{c}\text { Number of } \\
\text { Fin Sheets }\end{array}$ & $\begin{array}{c}\text { Central } \\
\text { Rubber } \\
\text { Type }\end{array}$ & $\begin{array}{c}\text { Voltage } \\
(\mathbf{V})\end{array}$ & $\begin{array}{c}\text { Amplitude } \\
\text { setting }\end{array}$ & $\begin{array}{c}\text { Weight } \\
\text { conditions }\end{array}$ & $\begin{array}{c}\text { Time } \\
(\mathbf{s})\end{array}$ & $\begin{array}{c}\text { Speed } \\
(\mathbf{c m} / \mathbf{s})\end{array}$ \\
\hline 3 & White & 22.1 & D6 & Normal & 8.39 & 35.8 \\
\hline 3 & Thin & 22.4 & D6 & Normal & 8.74 & 34.3 \\
\hline 3 & Thin & 22.2 & D8 & Normal & 9.32 & 32.12 \\
\hline 3 & Thin & 22.1 & D8 & Normal & 9.68 & 31.0 \\
\hline 3 & Thin & 22.0 & D8 & Heavy & 10.52 & 28.5 \\
\hline 3 & Thin & 22.3 & D8 & Heavy & 10.54 & 28.5 \\
\hline 3 & Thin & 22.2 & D4 & Normal & 10.77 & 27.9 \\
\hline
\end{tabular}


Table 2. Open-water testing with initial craft speed

\begin{tabular}{|c|c|c|c|c|c|c|}
\hline $\begin{array}{c}\text { Number of } \\
\text { Fin Sheets }\end{array}$ & $\begin{array}{c}\text { Central } \\
\text { Rubber } \\
\text { Type }\end{array}$ & $\begin{array}{c}\text { Voltage } \\
(\mathbf{V})\end{array}$ & $\begin{array}{c}\text { Amplitude } \\
\text { setting }\end{array}$ & $\begin{array}{c}\text { Weight } \\
\text { conditions }\end{array}$ & $\begin{array}{c}\text { Time } \\
(\mathbf{s})\end{array}$ & $\begin{array}{c}\text { Speed } \\
(\mathbf{c m} / \mathbf{s})\end{array}$ \\
\hline 3 & White & 21.9 & D6 & Normal & 5.73 & 34.9 \\
\hline 3 & White & 22.0 & D6 & Heavy & 7.28 & 27.5 \\
\hline 3 & White & 22.0 & D6 & Heavy & 7.39 & 27.1 \\
\hline
\end{tabular}




\section{Figures}

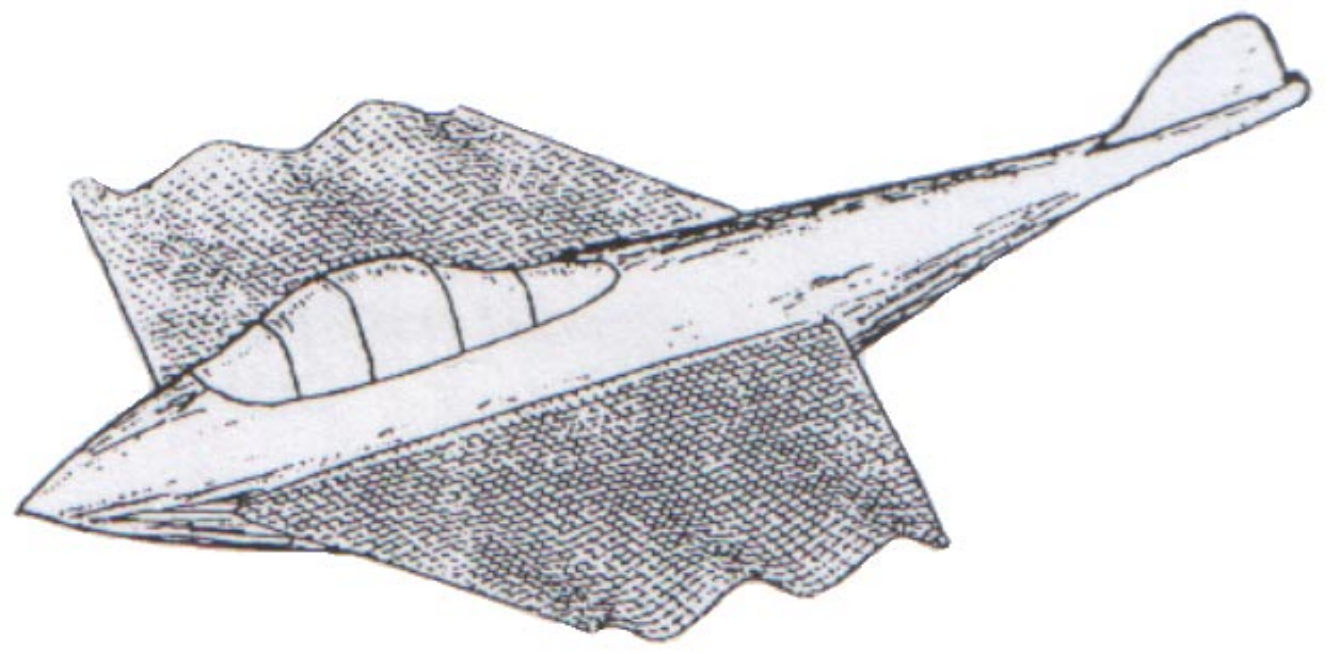

Fig. 1. On the proposed use of wedge elastic waves for propulsion of a small submarine [1]; the localised flexural waves propagate along the tips of the horizontal fins (wings), their energy being concentrated away from the main body. 


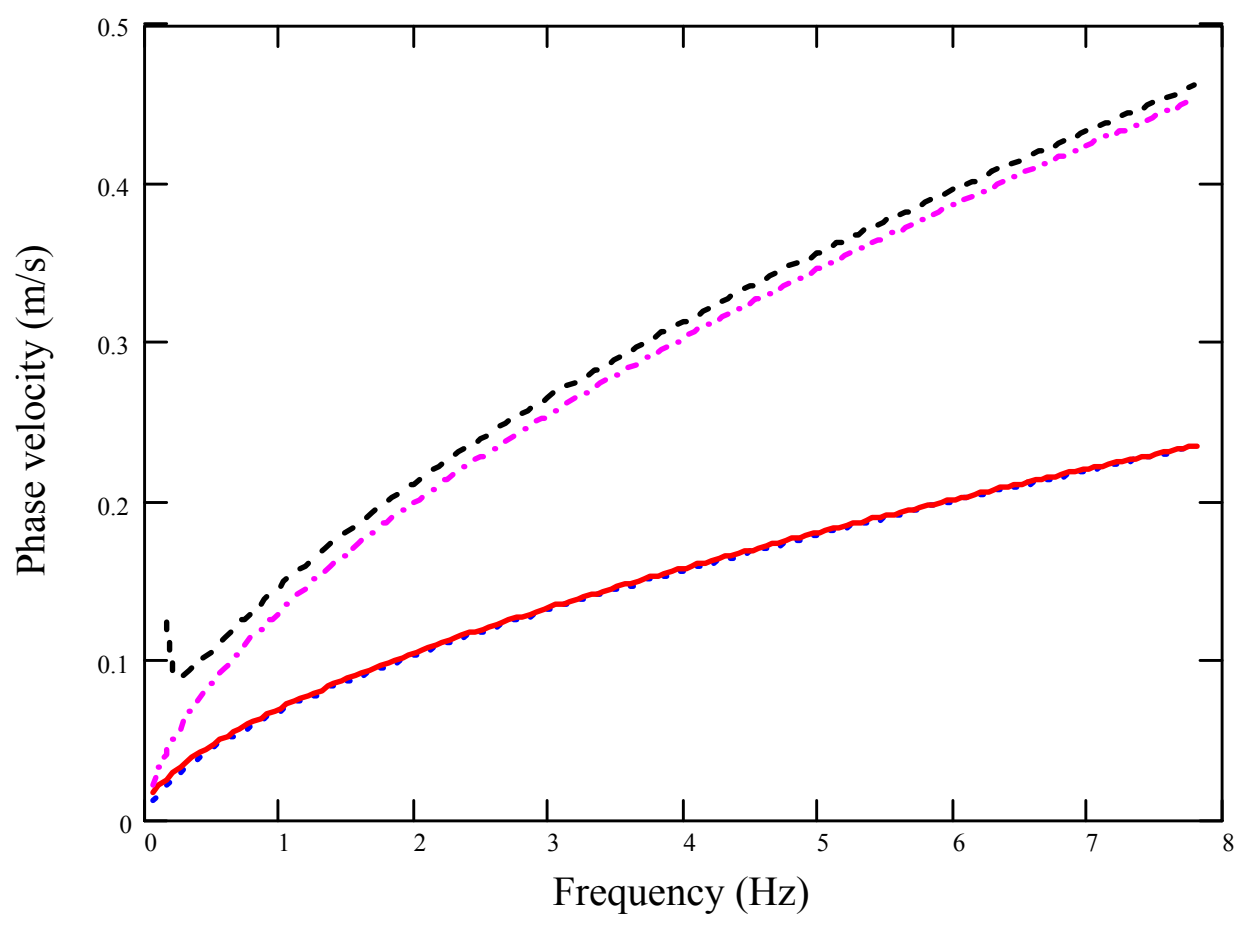

Fig. 2. Phase velocities of the lowest order flexural mode in two 'clamped-free' waterloaded rubber strips as functions of frequency; both strips are of the same width $H$ $=150 \mathrm{~mm}$, but have different values of thickness: $h=1 \mathrm{~mm}$ (solid curve) and $h=$ $3 \mathrm{~mm}$ (dashed curve); for comparison, the velocities for infinite plates are shown for $h=1 \mathrm{~mm}$ (dotted curve, which practically coincides with the solid curve) and for $h$ $=3 \mathrm{~mm}$ (dash-dotted curve). 


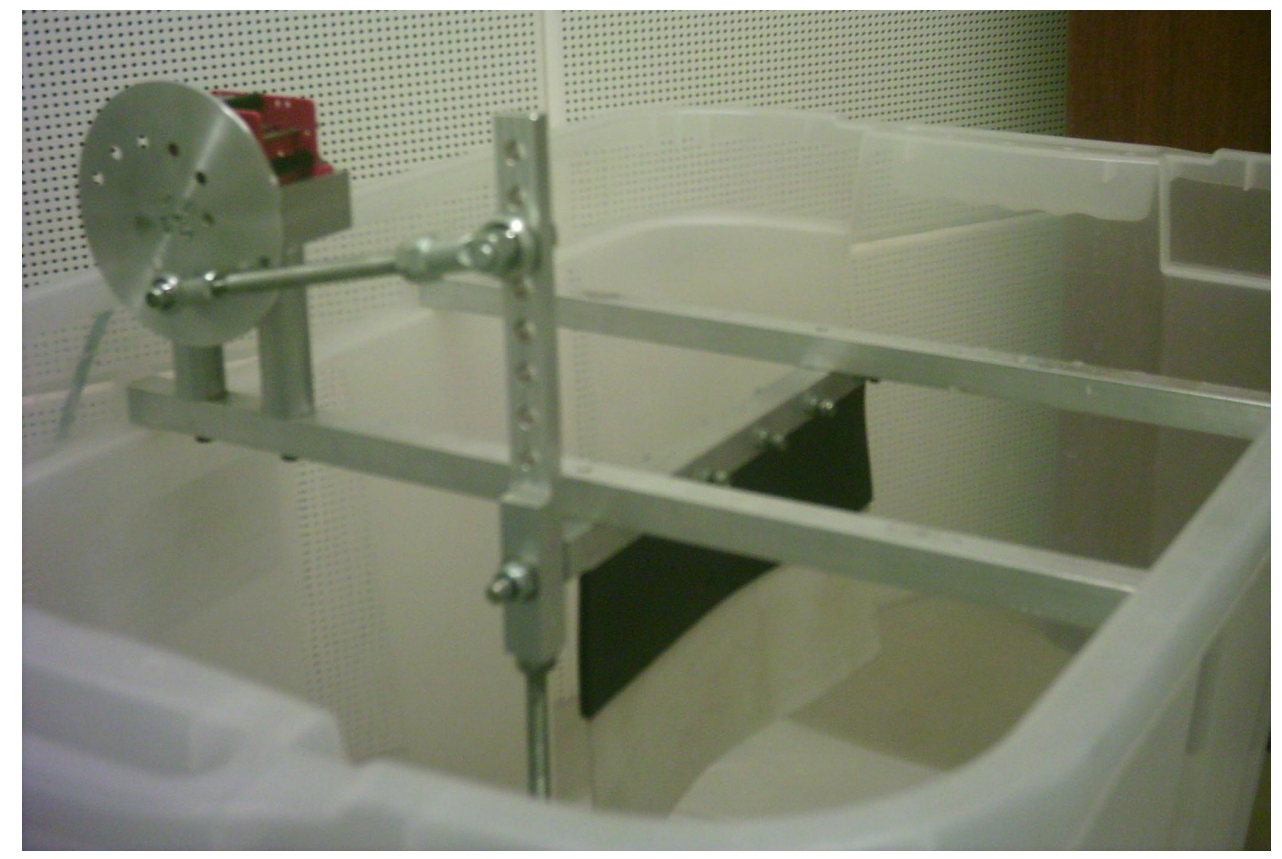

Fig. 3. View of the assembled propulsion rig in a an empty water tank used for in-tank testing: the main propulsive plate made of white rubber is stiffened by two narrower plates of black rubber; holes drilled on the motor disk are used for changing amplitudes of plate vibration. 


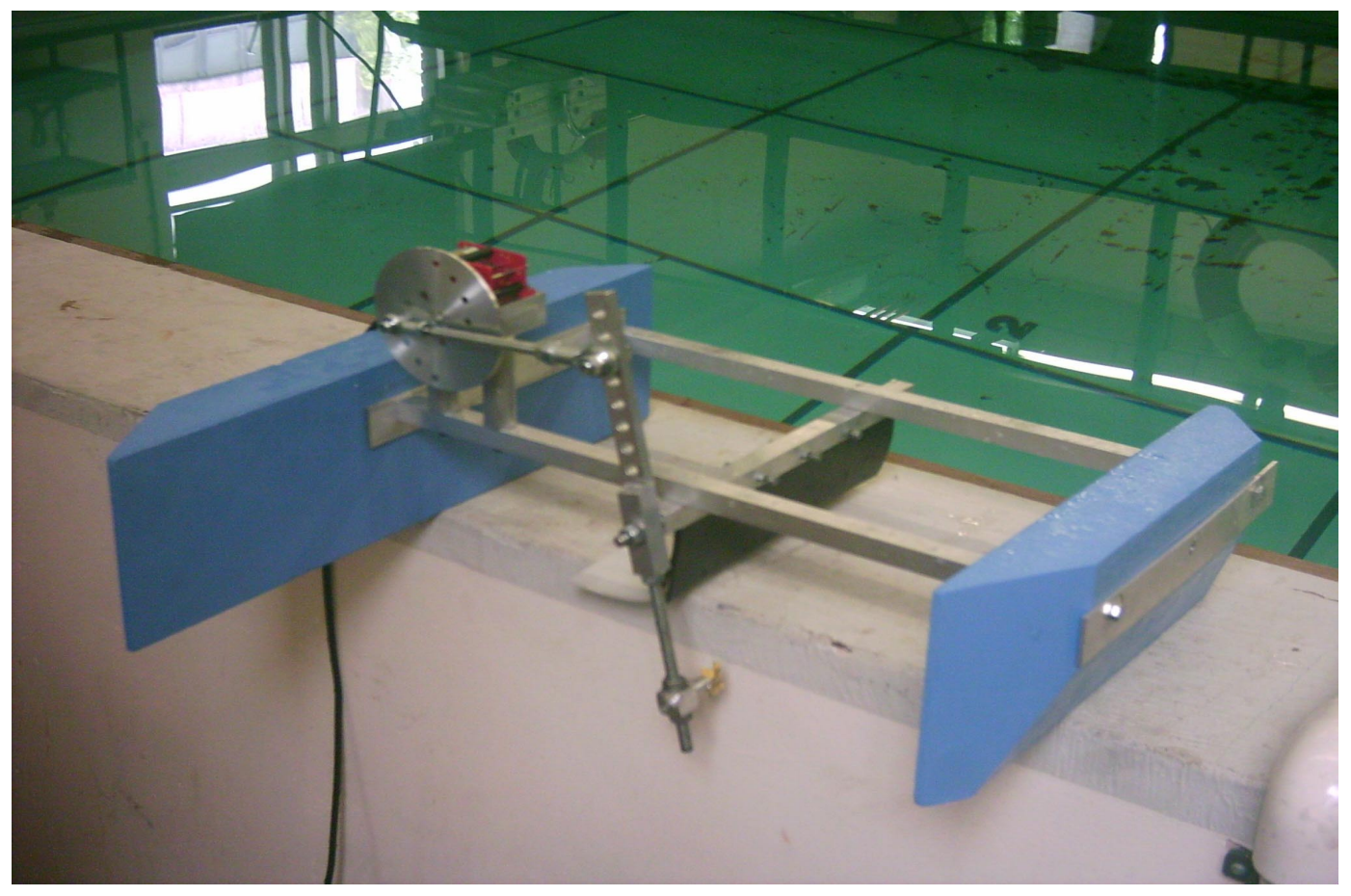

Fig. 4. View of the assembled catamaran with wedge wave propulsive system before its testing in open water. 


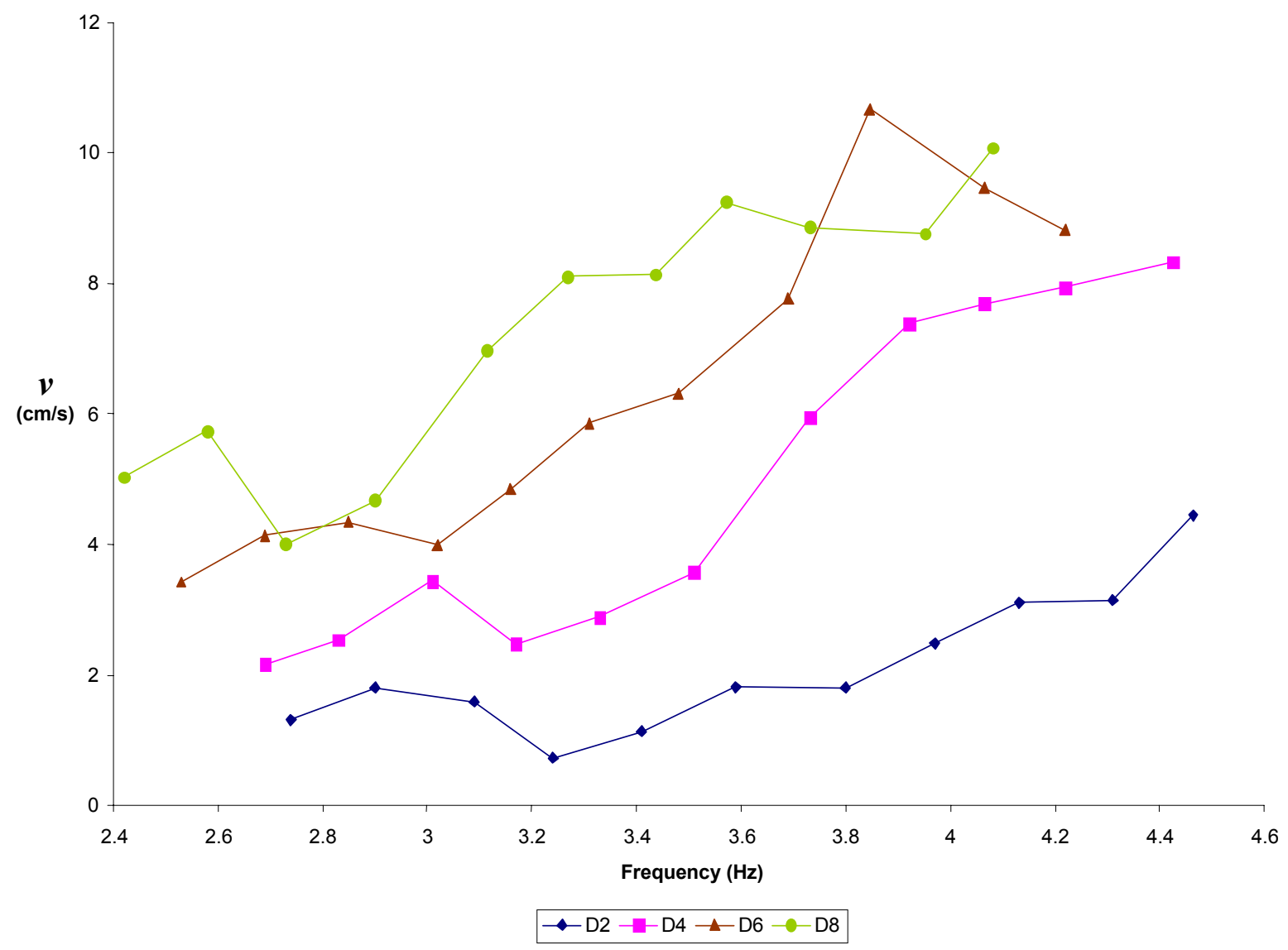

Fig. 5. Recorded speed $v$ of the flow generated in a water tank by a localised wedge elastic wave propagating along the rubber plate as function of frequency for different values of the wave displacement amplitudes; the amplitudes are identified by the locations of the holes on the motor disk: holes D2, D4, D6 and D8 correspond to the displacement amplitudes of $1.8,2.2,2.8$ and $3.2 \mathrm{~cm}$ respectively. 


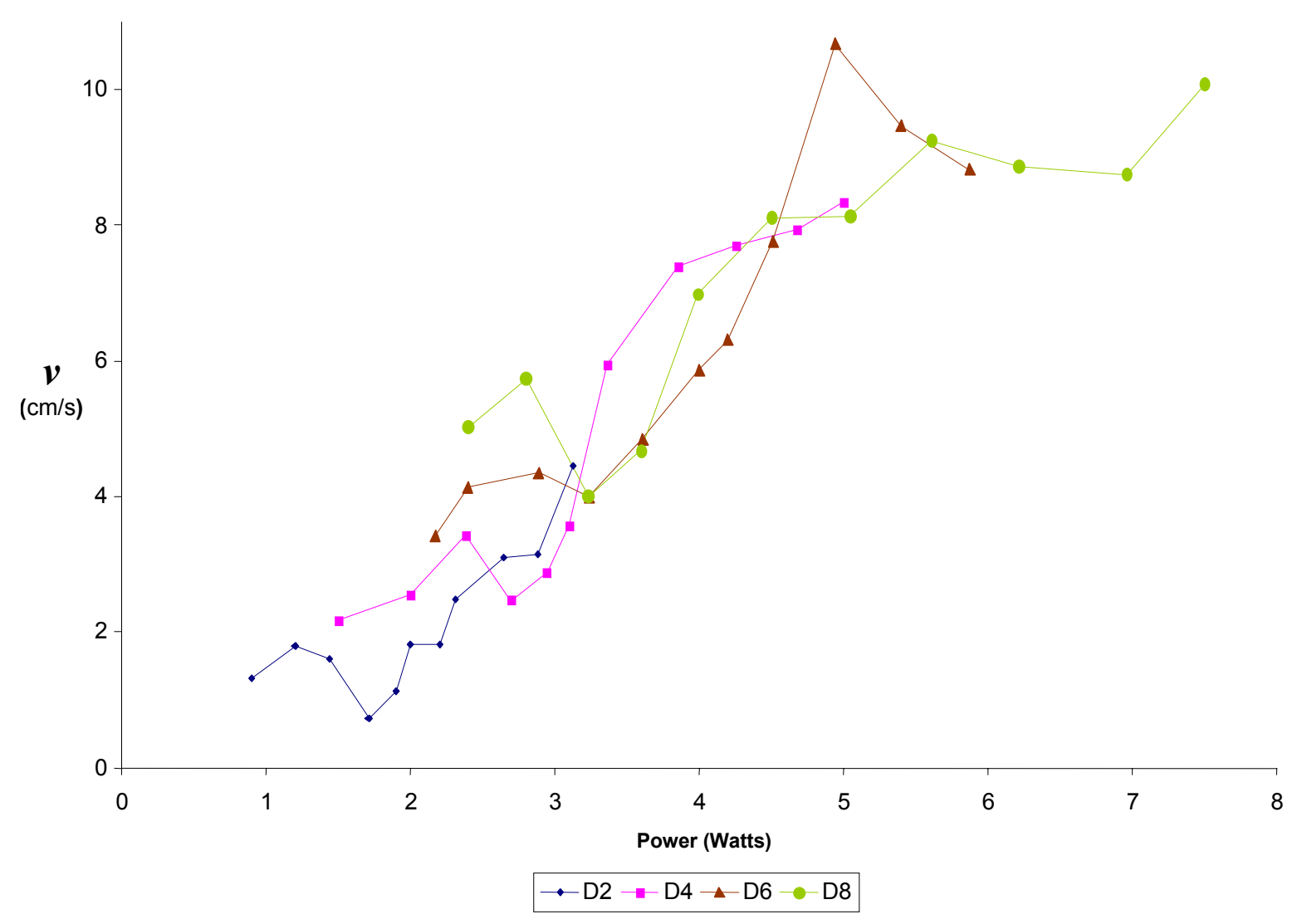

Fig. 6. Recorded speed $v$ of the flow generated in a water tank by a localised wedge elastic wave propagating along the rubber plate as function of input electric power for different values of the wave displacement amplitudes; the amplitudes are identified by the locations of the holes on the motor disk: holes D2, D4, D6 and D8 correspond to the displacement amplitudes of $1.8,2.2,2.8$ and $3.2 \mathrm{~cm}$ respectively. 


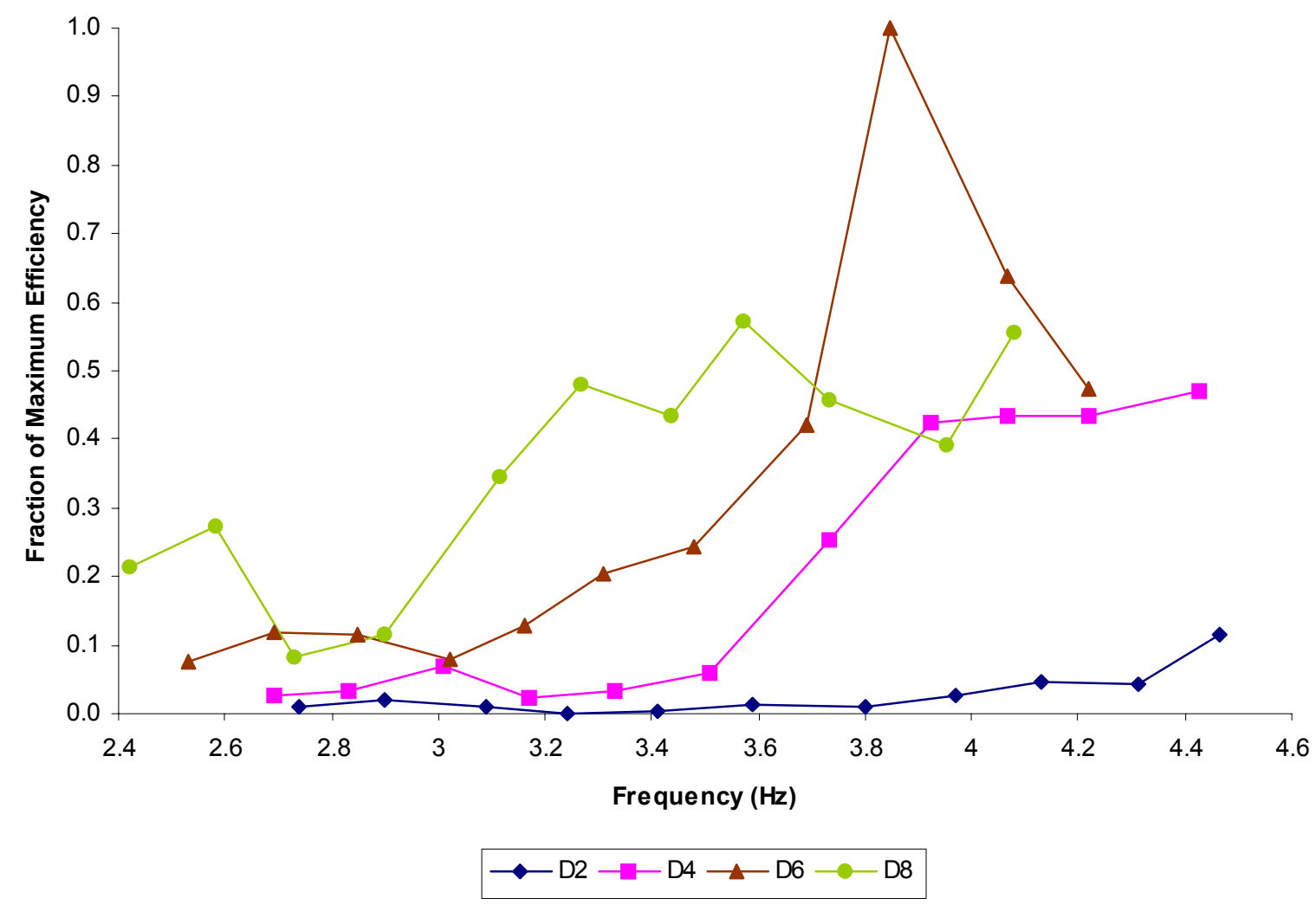

Fig. 7. Fraction of maximum efficiency of the process of flow generation in a water tank by a localised wedge elastic wave propagating along the rubber plate as function of frequency for different values of the wave displacement amplitudes; the amplitudes are identified by the locations of the holes on the motor disk: holes D2, D4, D6 and D8 correspond to the displacement amplitudes of $1.8,2.2,2.8$ and $3.2 \mathrm{~cm}$ respectively. 


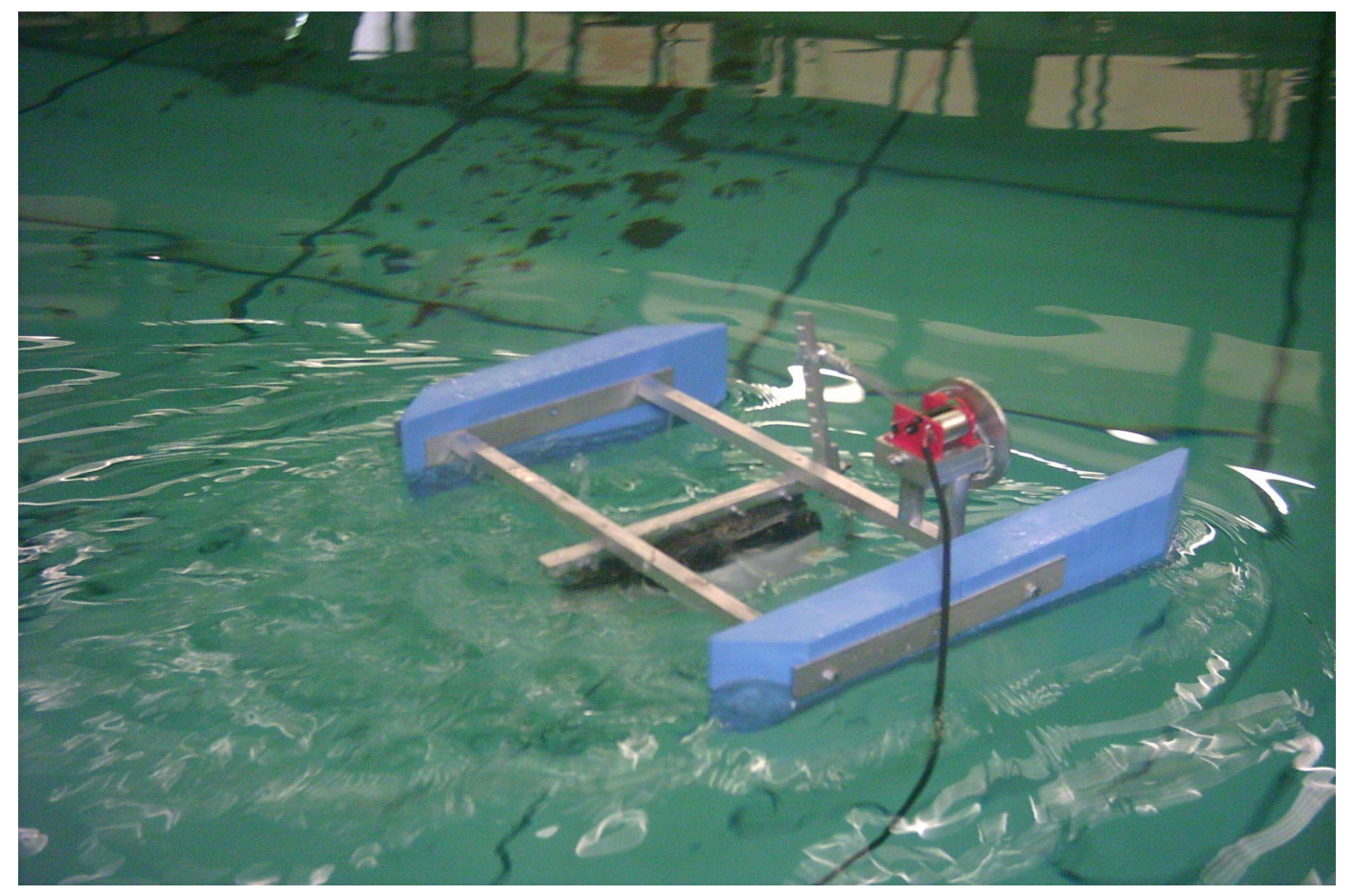

Fig. 8. View of the moving catamaran with wedge wave propulsive system during its testing in open water. 